\title{
Concurrent and Adjuvant Temozolomide for Newly Diagnosed Grade III Gliomas without 1p/19q Co-deletion: A Randomized, Open-Label, Phase 2 Study (KNOG-1101 Study)
}

\begin{tabular}{|c|}
\hline 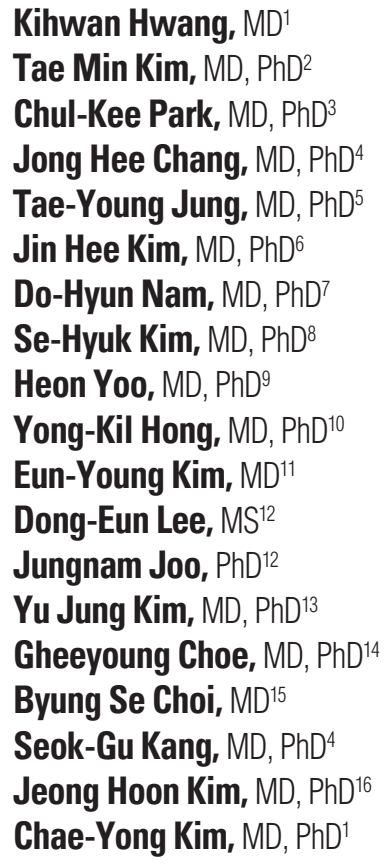 \\
\hline
\end{tabular}

${ }^{*}$ A list of author's affiliations appears at the end of the paper.

\footnotetext{
Correspondence: Jeong Hoon Kim, MD, PhD Department of Neurological Surgery, Asan Medical Center, University of Ulsan College of Medicine, 88 Olympic-ro 43-gil, Songpa-gu, Seoul 05505, Korea

Tel: 82-2-3010-3559

Fax: 82-2-476-6738

E-mail: jhkim1@amc.seoul.kr

Co-correspondence: Chae-Yong Kim, MD, PhD Department of Neurosurgery, Seoul National University Bundang Hospital, Seoul National University College of Medicine, 82 Gumi-ro 173 Beon-gil, Bundang-gu, Seongnam 13620, Korea Tel: $82-31-787-7165$

Fax: 82-31-787-4097

E-mail: chaeyong@snu.ac.kr

Received July 21, 2019

Accepted October 26, 2019

Published Online October 28, 2019
}

\begin{abstract}
Purpose
We investigated the efficacy of temozolomide during and after radiotherapy in Korean adults with anaplastic gliomas without 1p/19q co-deletion.

\section{Materials and Methods}

This was a randomized, open-label, phase 2 study and notably the first multicenter trial for Korean grade III glioma patients. Eligible patients were aged 18 years or older and had newly diagnosed non-co-deleted anaplastic glioma with an Eastern Cooperative Oncology Group performance status of 0-2. Patients were randomized 1:1 to receive radiotherapy alone (60 Gy in 30 fractions of $2 \mathrm{~Gy}$ ) (control group, $\mathrm{n}=44$ ) or to receive radiotherapy with concurrent temozolomide $\left(75 \mathrm{mg} / \mathrm{m}^{2} /\right.$ day) followed by adjuvant temozolomide (150-200 $\mathrm{mg} / \mathrm{m}^{2} /$ day for 5 days during six 28-day cycles) (treatment group, $\mathrm{n}=40$ ). The primary endpoint was 2-year progression-free survival (PFS). Seventy patients $(83.3 \%)$ were available for the analysis of the isocitrate dehydrogenase 1 gene (IDH1) mutation status.
\end{abstract}

\section{Results}

The two-year PFS was $42.2 \%$ in the treatment group and $37.2 \%$ in the control group. Overall survival (OS) did not reach to significant difference between the groups. In multivariable analysis, age was a significant risk factor for PFS (hazard ratio [HR], 2.08; 95\% confidence interval [Cl], 1.04 to 4.16). The IDH1 mutation was the only significant prognostic factor for PFS (HR, 0.28; 95\% Cl, 0.13 to 0.59) and OS (HR, 0.19; 95\% Cl, 0.07 to 0.50). Adverse events over grade 3 were seen in 16 patients (40.0\%) in the treatment group and were reversible.

\section{Conclusion}

Concurrent and adjuvant temozolomide in Korean adults with newly diagnosed nonco-deleted anaplastic gliomas showed improved 2-year PFS. The survival benefit of this regimen needs further analysis with long-term follow-up at least more than 10 years.

\section{Key words}

Anaplastic glioma, 1p/19q co-deletion, Temozolomide,

Chemotherapy, Adjuvant treatment 


\section{Introduction}

Anaplastic (i.e., World Health Organization [WHO] grade III) gliomas account for approximately $20 \%$ of adult gliomas [1]. While surgery and radiotherapy may prolong life, prognosis remains dismal, with a median survival of 2-5 years [2-4]. To date, despite inconclusive data, there has been growing evidence of improved survival that the addition of nitrosourea-based chemotherapy to radiotherapy could be beneficial for patients with WHO grade III gliomas [5]. In particular, adjuvant chemotherapy may be effective in $1 \mathrm{p} /$ $19 q$ co-deleted anaplastic oligodendrogliomas [6,7]. Two large randomized phase III trials of radiotherapy with or without adjuvant procarbazine, lomustine, and vincristine (PCV) demonstrated significant improvement in the progression-free survival (PFS) and near doubling of the overall survival (OS) to greater than 14 years in $1 p / 19 q$ co-deleted tumors treated with adjuvant radiotherapy and PCV [6,7]. However, patients with $1 \mathrm{p} / 19 \mathrm{q}$ non-co-deleted tumors may experience worse prognosis and faster tumor growth, requiring further attention. Although some studies suggested that $1 p / 19 q$ non-co-deleted tumors benefited from early chemotherapy, optimal indications, dosing regimens, or survival analyses remain controversial [1]. Therefore, we focused on these conditions and analyses and on how the CATNON trial was distinguishable from other studies [8].

Separately, the role of chemotherapy for gliomas has been reappraised with the development of temozolomide, and clear benefits were found when temozolomide was added to radiotherapy to treat newly diagnosed glioblastomas [9]. Further investigation is now being directed toward unveiling its optimal indications, leading researchers to explore whether radiation plus temozolomide would benefit patients with anaplastic gliomas. Some of our authors have investigated the potential survival benefit and safety of concurrent chemoradiotherapy (CCRT) with temozolomide followed by adjuvant temozolomide for WHO grade III gliomas $[10,11]$. In these studies, however, the role of molecular markers, such as chromosome $1 \mathrm{p} / 19 \mathrm{q}$ co-deletion or $\mathrm{O}^{6}$-methylguanine-DNA methyltransferase (MGMT) promoter methylation, could not be determined because of the small sample size. These results prompted the authors to conduct a multiinstitutional trial.

We investigated the efficacy of temozolomide during and after radiotherapy in Korean adult patients with newly diagnosed WHO grade III gliomas without the $1 \mathrm{p} / 19 \mathrm{q}$ co-deletion. In this study, the authors studied the role of temozolomide in patients who were expected to have worse prognoses according to the status of chromosome $1 \mathrm{p} / 19 \mathrm{q}$, i.e., $1 \mathrm{p} / 19 \mathrm{q}$ non-co-deleted tumors. The treatment group (surgery plus CCRT with temozolomide followed by six cycles of adjuvant temozolomide) was compared to the control group (surgery plus radiotherapy alone) in terms of the efficacy and safety of temozolomide.

\section{Materials and Methods}

\section{Study design and patients}

The KOREANA study was a multicenter study performed by the Korean Neuro-Oncology Group of Korean Society for Neuro-Oncology (KSNO). The trial was a randomized, openlabel, phase 2 study to evaluate the efficacy between only radiotherapy versus combination therapy with radiotherapy and temozolomide in Korean adult patients with newly diagnosed supratentorial grade III gliomas without the $1 \mathrm{p} /$ $19 \mathrm{q}$ co-deletion. The protocol was registered at clinicaltrials.gov (NCT01534845).

Patients with newly diagnosed, histologically proven supratentorial anaplastic gliomas, i.e., WHO grade III glioma without the $1 p / 19 q$ co-deletion was eligible to participate in this study. The inclusion criteria were as follows: diagnosis of anaplastic gliomas according to the 2007 WHO criteria; age $\geq 18$ years; Eastern Cooperative Oncology Group performance status of 0 or 1 ; stable or decreasing dose of steroids for $\geq 5$ days prior to randomization; recursive partitioning analysis classification of III, IV, or V; and adequate hematologic, renal, and hepatic functions, including an absolute neutrophil count $>1,500 / \mu \mathrm{L}$, platelet count $\geq 100,000 / \mu \mathrm{L}$, serum creatinine $<1.7 \mathrm{mg} / \mathrm{dL}$, bilirubin level $\leq 2.0 \mathrm{mg} / \mathrm{dL}$, and aspartate aminotransferase/alanine aminotransferase $\leq 2.5 \times$ the upper limit of the normal range of each institution.

The exclusion criteria consisted of having prior chemotherapy within the last 5 years, having prior radiotherapy of the head and neck area, receiving concurrent investigational agents or having received an investigational agent within 30 days prior to randomization, having planned surgery for other diseases, having a history of malignancy with the exception of cervical carcinoma in situ or basal cell carcinoma of the skin, being pregnant or lactating, refusing to use effective contraception, having concurrent illness that would interfere with the prescribed treatment, and being unable to undergo gadolinium-based magnetic resonance imaging (MRI).

\section{Treatment schema}

At enrollment, the diagnosis of anaplastic glioma had to be confirmed at each institution. In addition, the tumor material was submitted for assessment of the $1 \mathrm{p} / 19 \mathrm{q}$ co-dele- 
tion status and review of pathology by pathologist (G.C.) at the central laboratory. The $1 \mathrm{p} / 19 \mathrm{q}$ co-deletion status was assessed by fluorescent in situ hybridization [12]. After identifying the status of the $1 \mathrm{p} / 19 \mathrm{q}$ co-deletion, patients were screened for inclusion and then assigned in equal numbers to receive CCRT with temozolomide followed by adjuvant temozolomide (treatment group [arm A]) or to receive radiotherapy alone (control group [arm B]). The clinical data were collected and validated with a web-based clinical research management platform (Velos Inc., Fremont, CA).

All patients received fractionated focal irradiation in daily fractions of 2 Gy given 5 days per week for 6 weeks, for a total of $60 \mathrm{~Gy}$. When given concurrently with radiotherapy, temozolomide was given daily from the first to the last day of radiotherapy, including on non-radiotherapy weekend days, at a dose of $75 \mathrm{mg} / \mathrm{m}^{2}$ for a maximum of 6 weeks. As adjuvant chemotherapy, temozolomide was started 4 weeks after completion of radiotherapy for a maximum of six cycles. Patients received $150 \mathrm{mg} / \mathrm{m}^{2}$ temozolomide on days $1-5$ of the first cycle and $200 \mathrm{mg} / \mathrm{m}^{2}$ on days $1-5$ of subsequent cycles if no or minor toxicity was seen during the first cycle, and dose modifications could be made as described elsewhere [9]. Treatment after disease progression, the rescue regimen will be administered: the use of temozolomide was suggested for patients who had been assigned to receive radiotherapy alone.

\section{Response assessment}

Patients were followed up weekly during radiotherapy, every 2 weeks during adjuvant temozolomide treatment, and every 3 or 4 months after the completion of all treatment. Tumors were assessed with MRI and steroid dose. MRI was performed 4 weeks after the end of radiotherapy and every 3 months until the second year and every 6 months thereafter until disease progression. Radiographic response is determined in comparison to the tumor measurement obtained at pretreatment baseline measurements as a reference and is categorized into four groups using new criteria proposed by the Response Assessment in Neuro-Oncology working group [13]. Patients with possible pseudoprogression were kept on current treatment and re-evaluated 4 weeks thereafter to clarify the response. Steroid dose was reviewed at every outpatient visit. Clinical evaluation with adverse events was scored with the National Cancer Institute Common Toxicity Criteria for Adverse Event ver. 4.0.

The evaluation of health-related quality of life was assessed using the European Organization for Research and Treatment of Cancer quality-of-life questionnaire (QLQ-C30) and brain module (QLQ-BN20). Questionnaires were completed on paper at baseline (prior to randomization) and subsequently at all visits when MRI was performed. Data on health-related quality of life will be reported separately.

\section{Outcomes}

The primary endpoint was 2-year PFS. Landmark PFS was also analyzed. PFS was calculated from the date of randomization to the date of first tumor progression or death whichever was earlier, with censoring of patients who were lost to follow-up. Two-year PFS was defined as the survival rate at the second year from randomization.

The second endpoints were overall PFS and OS adjusted for stratification factors, health-related quality of life outcomes, and adverse events. OS was defined as the time from randomization to the date of death from any cause.

\section{Statistical analysis}

Baseline characteristics, details of treatments and adverse events were expressed as the mean with standard deviation for continuous variables and the frequency with percentage for categorical variables. The comparisons between arm A and arm B were carried out using Student's t test, Pearson's chi-square or Fisher exact test, as appropriate. The survival curves were estimated using the Kaplan-Meier method, and the difference between survival curves was evaluated using the log-rank test. The Kaplan-Meier estimates at fixed time points $(3,6,12,18$, and 24 months for PFS and 12, 24, and 60 months for OS) were compared based on the $\mathrm{Z}$ test. The $\mathrm{Z}$ test statistics used variance estimators calculated with Greenwood's formula. To explore the prognostic factors for PFS and OS, univariable Cox proportional hazards models were performed. A multivariable Cox proportional hazard model with a backward elimination criterion of $p>0.2$ was applied. The 2-year PFS, which was the primary outcome of the study, was considered statistically significant when the one-sided $\mathrm{p}$-value was less than 0.2. Otherwise, the results were regarded as statistically significant when the two-sided p-value was less than 0.05 . Statistical analyses were performed using SAS ver. 9.4 (SAS Institute Inc., Cary, NC).

\section{Ethical statement}

A total of 14 Korean institutions participated in this study with ethics approval from their institutional review boards before enrollment started. All patients gave written informed consent according to national guidelines. Patients were not compensated for their participation. 
Table 1. Baseline characteristics of patients

\begin{tabular}{|c|c|c|c|c|}
\hline & $\begin{array}{l}\text { Total } \\
(\mathrm{n}=84)\end{array}$ & $\begin{array}{l}\text { CCRT with temozolomide plus } \\
\text { adjuvant temozolomide }(n=40)\end{array}$ & $\begin{array}{l}\text { Radiotherapy } \\
\text { alone }(n=44)\end{array}$ & p-value \\
\hline Age (yr) & $44.7 \pm 13.2$ & $44.7 \pm 13.3$ & $44.8 \pm 13.2$ & $0.980^{\mathrm{a})}$ \\
\hline \multicolumn{5}{|l|}{ Sex } \\
\hline Male & $46(54.8)$ & $23(57.5)$ & $23(52.3)$ & $0.631^{b)}$ \\
\hline Female & $38(45.2)$ & $17(42.5)$ & $21(47.7)$ & \\
\hline \multicolumn{5}{|l|}{ ECOG performance status } \\
\hline 0 & $34(40.5)$ & $12(30.0)$ & $22(50.0)$ & $0.143^{c)}$ \\
\hline 1 & $44(52.4)$ & $24(60.0)$ & $20(45.5)$ & \\
\hline 2 & $6(7.14)$ & $4(10.0)$ & $2(4.5)$ & \\
\hline \multicolumn{5}{|l|}{ Extent of tumor resection } \\
\hline Gross total resection & $37(44.1)$ & $17(42.5)$ & $20(45.5)$ & $0.681^{b)}$ \\
\hline Subtotal resection & $17(20.2)$ & $9(22.5)$ & $8(18.2)$ & \\
\hline Partial resection & $14(16.7)$ & $5(12.5)$ & $9(20.5)$ & \\
\hline Biopsy only & $16(19.1)$ & $9(22.5)$ & $7(15.9)$ & \\
\hline \multicolumn{5}{|l|}{$M G M T$ promotor methylation } \\
\hline Unmethylation & $42(64.6)$ & $21(65.6)$ & $21(63.6)$ & $0.867^{\mathrm{b})}$ \\
\hline Methylation & $23(35.4)$ & $11(34.4)$ & $12(36.4)$ & \\
\hline Missing & 19 & 8 & 11 & \\
\hline \multicolumn{5}{|l|}{ IDH1 mutation } \\
\hline Negative & $44(62.9)$ & $22(64.7)$ & $22(61.1)$ & $0.756^{\mathrm{b})}$ \\
\hline Positive & $26(37.1)$ & $12(35.3)$ & $14(38.9)$ & \\
\hline Missing & 14 & 6 & 8 & \\
\hline Follow-up duration, median (range, mo) & $44.9(2.8-71.1)$ & $52.9(3.6-69.7)$ & $42.1(2.8-71.1)$ & $0.209^{\mathrm{d})}$ \\
\hline
\end{tabular}

Values are presented as mean \pm standard deviation or number (\%) unless otherwise indicated. CCRT, concurrent chemoradiotherapy; ECOG, Eastern Cooperative Oncology Group; MGMT, $\mathrm{O}^{6}$-methylguanine-DNA methyltransferase; IDH1, isocitrate dehydrogenase 1. ${ }^{\mathrm{a}}$ Student's $\mathrm{t}$ test, ${ }^{\mathrm{b})} \mathrm{Chi}$-square test, ${ }^{\mathrm{c}}$ Fisher exact test, ${ }^{\mathrm{d}}$ Log-rank test.

Table 2. Details and intensities of the treatment

\begin{tabular}{|c|c|c|c|}
\hline & $\begin{array}{l}\text { CCRT with temozolomide } \\
\text { plus adjuvant temozolomide }\end{array}$ & $\begin{array}{l}\text { Radiotherapy } \\
\text { alone }\end{array}$ & p-value \\
\hline Total dose of radiotherapy (Gy) & $60.1 \pm 1.3$ & $60.2 \pm 3.6$ & $0.771^{\text {a) }}$ \\
\hline Duration of radiotherapy (wk) & $6.3 \pm 0.9$ & $6.1 \pm 0.7$ & $0.188^{\mathrm{a})}$ \\
\hline Duration of temozolomide with CCRT (wk) & $5.7 \pm 0.9$ & - & - \\
\hline Duration of temozolomide after CCRT (cycle, missing $=2$ ) & $5.3 \pm 1.6$ & - & - \\
\hline \multicolumn{4}{|c|}{ Response of treatment at TMZ or after radiotherapy cycle 1 (missing=1) } \\
\hline Complete response & $2(5.1)$ & $1(2.3)$ & $0.869^{b)}$ \\
\hline Partial response & $9(23.1)$ & $12(27.3)$ & \\
\hline Stable disease & $22(56.4)$ & $24(54.6)$ & \\
\hline Progressive disease & $3(7.7)$ & $5(11.4)$ & \\
\hline Not assessed & $3(7.7)$ & $2(4.5)$ & \\
\hline \multicolumn{4}{|l|}{ Response of treatment at end of treatment (missing=19) } \\
\hline Complete response & 0 & $1(3.0)$ & $0.851^{\mathrm{c})}$ \\
\hline Partial response & $7(21.2)$ & $5(15.2)$ & \\
\hline Stable disease & $19(57.6)$ & $20(60.6)$ & \\
\hline Progressive disease & $6(18.2)$ & $6(18.2)$ & \\
\hline Not assessed & $1(3.0)$ & $1(3.0)$ & \\
\hline
\end{tabular}

CCRT, concurrent chemoradiotherapy; TMZ, temozolomide. ${ }^{a)}$ Student's $t$ test, ${ }^{\mathrm{b})}$ Chi-square test, ${ }^{\mathrm{c}}$ Fisher exact test. 


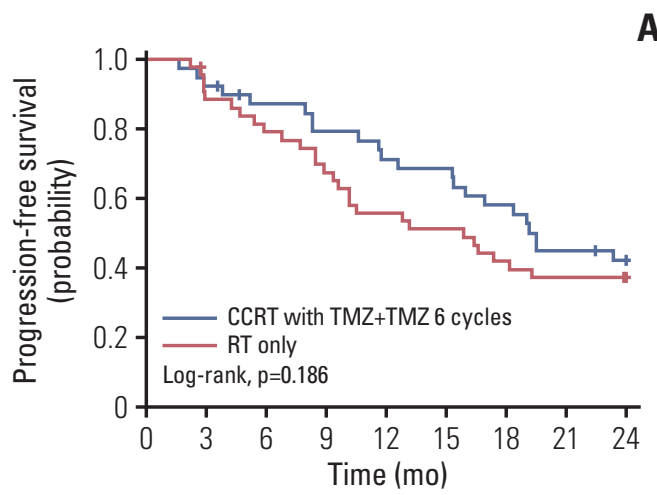

A

Fig. 1. Kaplan-Meier curve of progression-free survival (A) and overall survival (B). CCRT, concurrent chemoradiotherapy; TMZ, temozolomide; RT, radiotherapy.

Table 3. Progression-free survival and overall survival according to treatment

\begin{tabular}{|c|c|c|c|}
\hline & $\begin{array}{l}\text { CCRT with temozolomide } \\
\text { plus adjuvant temozolomide }\end{array}$ & $\begin{array}{l}\text { Radiotherapy } \\
\text { alone }\end{array}$ & $p$-value ${ }^{a}$ \\
\hline \multicolumn{4}{|c|}{ Progression-free survival } \\
\hline Median (mo) & 19.5 & 15.9 & \\
\hline At $3 \mathrm{mo}(\%)$ & 92.5 & 90.7 & 0.773 \\
\hline At $6 \mathrm{mo}(\%)$ & 87.3 & 79.1 & 0.317 \\
\hline At $12 \mathrm{mo}(\%)$ & 71.4 & 55.8 & 0.139 \\
\hline At $18 \mathrm{mo}(\%)$ & 58.2 & 41.9 & 0.137 \\
\hline At $24 \mathrm{mo}(\%)$ & 42.2 & 37.2 & 0.652 \\
\hline \multicolumn{4}{|l|}{ Overall survival } \\
\hline Median (mo) & 43.7 & 36.7 & \\
\hline At $12 \mathrm{mo}(\%)$ & 84.0 & 95.2 & 0.100 \\
\hline At $24 \mathrm{mo}(\%)$ & 63.7 & 63.0 & 0.945 \\
\hline
\end{tabular}

CCRT, concurrent chemoradiotherapy. ${ }^{a}$ Landmark analysis ( $\mathrm{Z}$ test).

\section{Results}

\section{Patients and treatment}

From March 2012 to February 2015, 146 patients were screened, and 90 patients were randomly assigned to treatment groups. Six patients did not start treatment because of either consent withdrawal $(n=4)$ or disease progression $(n=2)$, and a total of 84 were included in the final analysis. There were 46 males $(54.8 \%)$ and 38 females $(45.2 \%)$. The mean age of patients was $44.7 \pm 13.2$ years. Fifty-four patients $(64.3 \%)$ underwent removal of over $75 \%$ of the tumor mass, including gross total removal or subtotal removal, and 30 patients $(35.8 \%)$ underwent partial removal or biopsy only. The results were available for the MGMT promoter methy- lation status for 65 of 84 patients $(77.4 \%)$ and the isocitrate dehydrogenase 1 gene (IDH1) mutation status for 70 of 84 patients $(83.3 \%)$ at the time of the preliminary analysis. Patients were randomly assigned to each treatment group according to the study protocol as follows: 44 patients received radiotherapy alone, and 40 patients received CCRT with temozolomide plus adjuvant temozolomide. The characteristics of patients in these two groups were well balanced at the baseline (Table 1).

In this trial, radiotherapy was completed in all but one of 84 patients $(1.2 \%)$ in whom it was started. Among 40 patients assigned to the CCRT with temozolomide plus adjuvant temozolomide group, 39 patients $(97.5 \%)$ completed radiotherapy with concurrent temozolomide. The mean therapeutic radiation dose was $60.1 \pm 1.3 \mathrm{~Gy}$, and the mean duration of temozolomide with CCRT was 5.7 \pm 0.9 weeks. After exclud- 


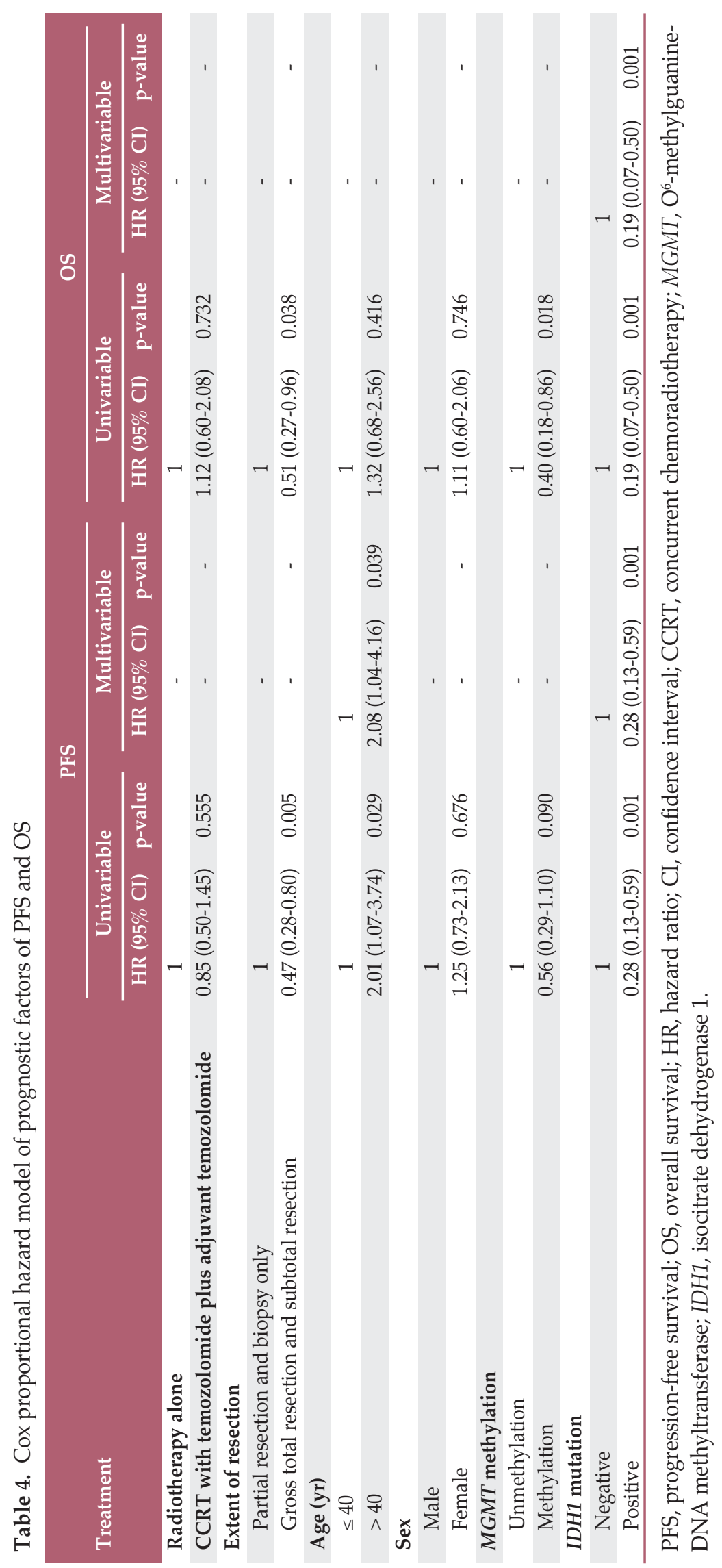



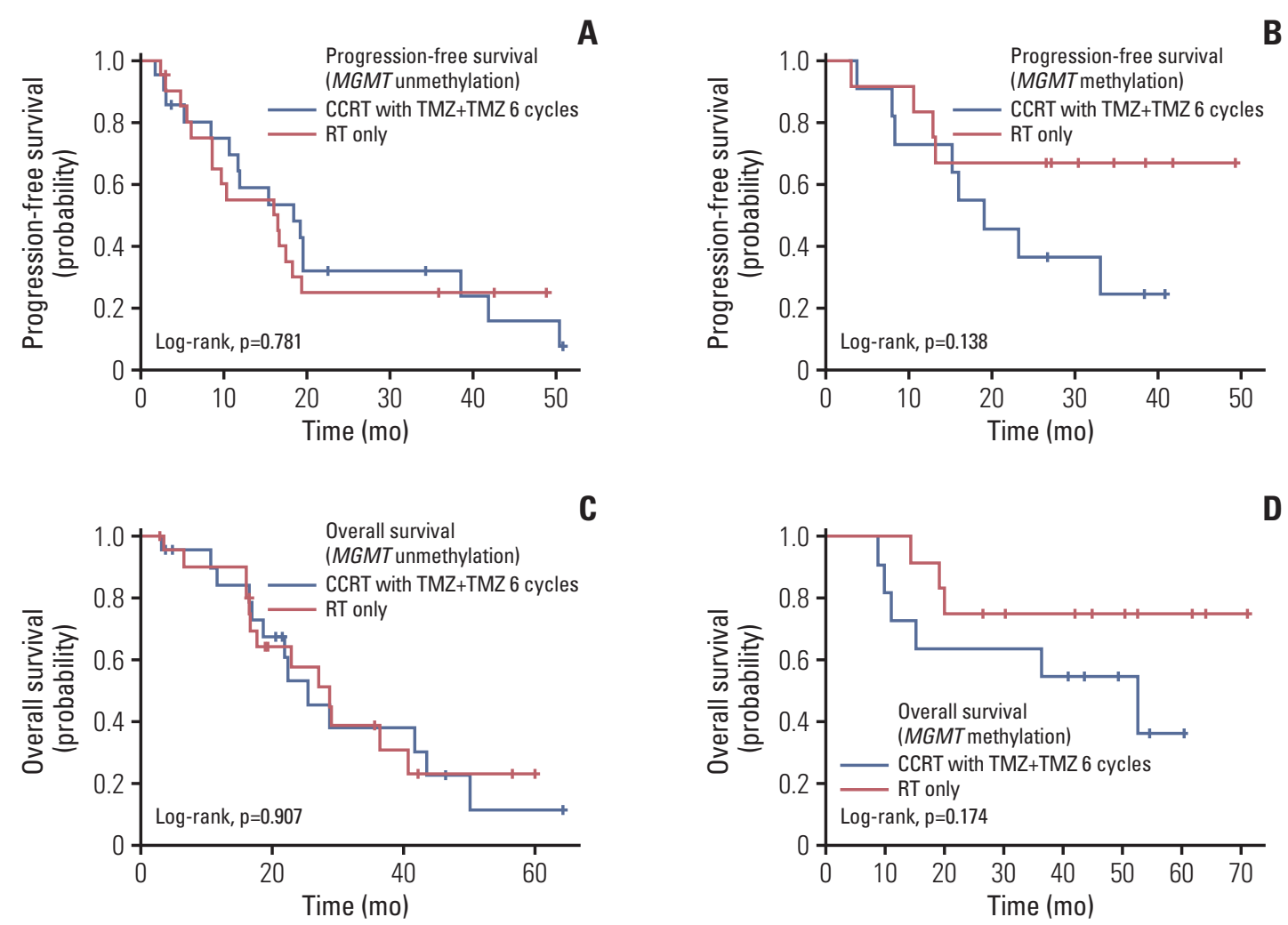

Fig. 2. Kaplan-Meier curve of progression-free survival (A, B) and overall survival (C, D) according to $\mathrm{O}^{6}$-methylguanineDNA methyltransferase (MGMT) promoter methylation status. CCRT, concurrent chemoradiotherapy; TMZ, temozolomide; $\mathrm{RT}$, radiotherapy.

ing one patient who progressed before starting adjuvant temozolomide, 32 of 38 patients $(84.2 \%)$ completed adjuvant temozolomide with a mean of $5.3 \pm 1.6$ cycles. Table 2 summarizes the details of the treatment delivered to the two treatment groups. There were no statistically significant differences in total radiation dose, duration of radiotherapy, or response to treatment between the treatment groups.

\section{Tumor control and survival}

After treatment, the objective response rate (complete response and partial response) and the tumor control rate (complete response, partial response, and stable disease) among patients who received radiotherapy alone were $18.8 \%$ and $81.3 \%$, respectively. For patients who received CCRT with temozolomide plus adjuvant temozolomide, the response rate and the tumor control rate were $21.9 \%$ and $81.3 \%$, respectively. The response to each treatment at the end of treatment showed no statistical significance (Table 2).

Kaplan-Meier survival curves of the patients according to the treatment are illustrated in Fig. 1. CCRT with temozolomide plus adjuvant temozolomide was associated with an improved 2-year PFS (log-rank $\mathrm{p}=0.186$ ), as we set one-sided type I error as 0.2 , but not with OS. For patients who received radiotherapy alone, the median PFS was 15.9 months, and the 2-year PFS was $37.2 \%$. The median OS was 36.7 months, and the 12- and 24-month OS rates were 95.2\% and 63.0\%, respectively. Among patients who received CCRT with temozolomide plus adjuvant temozolomide, the median PFS was 19.5 months, and the 2-year PFS was $42.2 \%$. The median OS was 43.7 months, and the 12- and 24-month OS rates were $84.0 \%$ and $63.7 \%$, respectively. The PFS and OS landmark values are presented in Table 3.

Prognostic factors, including the treatment, extent of resection, age, sex, methylation of the MGMT promoter, and IDH1 mutation were analyzed. In the univariable analysis, the extent of resection, age, and IDH1 mutation were significant prognostic factors for PFS. By the multivariable analysis, the hazard ratio (HR) for PFS adjusted for baseline stratification factors among patients with IDH1 mutation was 0.28 (95\% confidence interval [CI], 0.13 to $0.59 ; \mathrm{p}=0.001$ ). Age was a significant risk factor for PFS (HR, 2.08; 95\% CI, 1.04 to 4.16; $\mathrm{p}=0.039$ ). For OS, the significant prognostic factor in the univariable analysis was the extent of resection, the methylation 
A
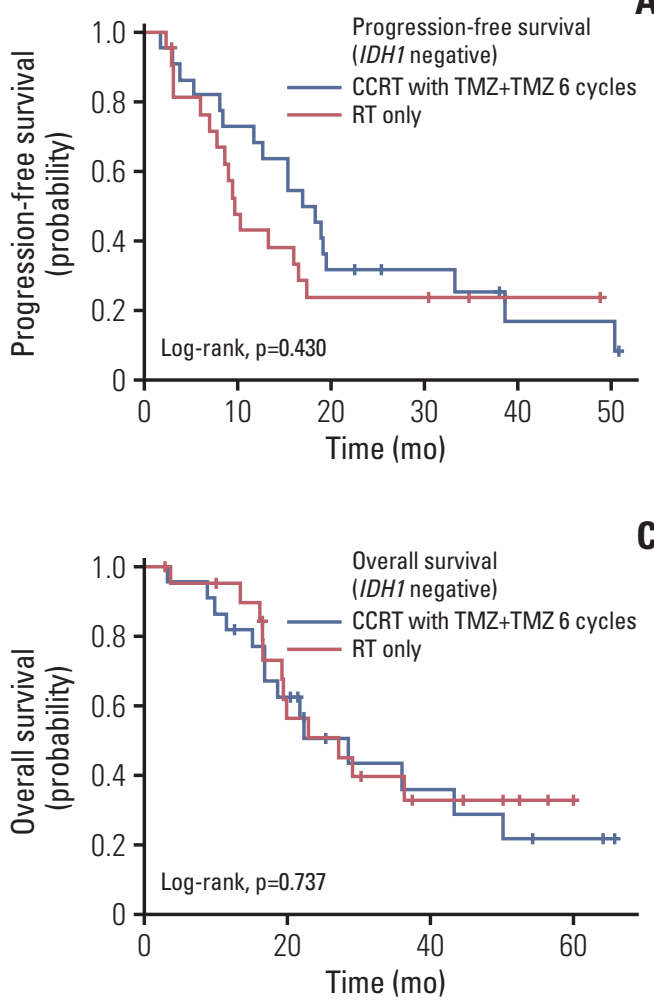

B

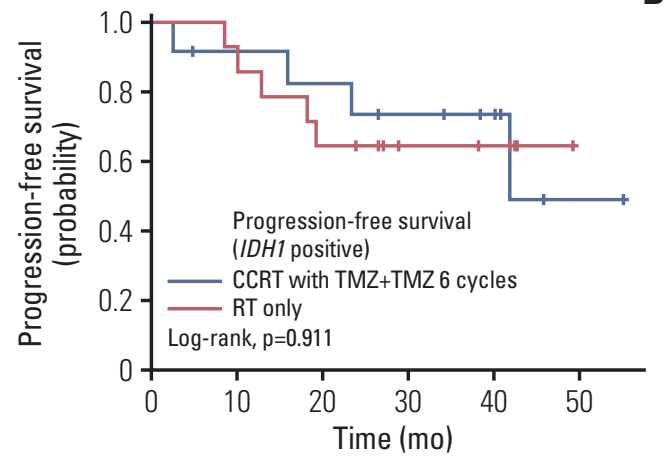

D

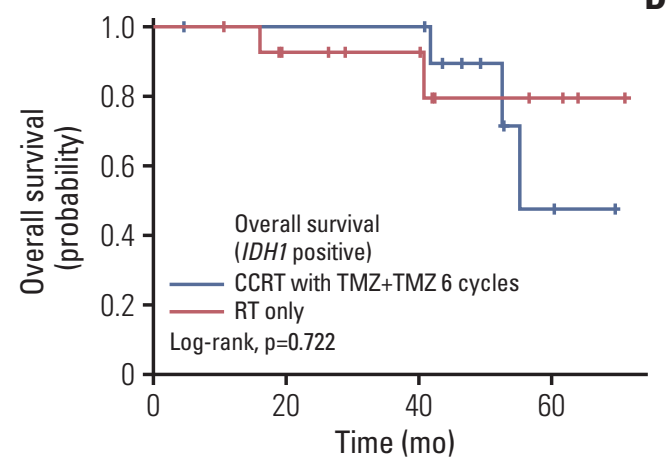

Fig. 3. Kaplan-Meier curve of progression-free survival (A, B) and overall survival (C, D) according to isocitrate dehydrogenase 1 (IDH1) mutation status. CCRT, concurrent chemoradiotherapy; TMZ, temozolomide; RT, radiotherapy.

of the MGMT promoter, and IDH1 mutation. However, IDH1 mutation was the only significant prognostic factor for OS (HR, $0.19 ; 95 \% \mathrm{CI}, 0.07$ to $0.50 ; \mathrm{p}=0.001$ ) by multivariable analysis (Table 4 ).

\section{Subgroup analysis according to genotypical characteris- tics}

Since we started this trial, mutations in IDH1 have been widely known to be associated with improved outcomes and incorporated into the 2016 WHO classification of glioma $[14,15]$. To clarify whether the presence of MGMT promoter methylation or IDH1 mutation could show differences in survival according to treatment, PFS and OS according to treatment and genotypical characteristics were analyzed. In a subgroup analysis, however, Kaplan-Meier survival curves showed no significant differences between both treatment groups according to the MGMT promoter methylation status (Fig. 2) or IDH1 mutation status (Fig. 3).

\section{Toxic effects}

There were 24 patients, 16 of 40 patients $(40.0 \%)$ in the treatment group and eight of 44 patients $(18.2 \%)$ in the control group, who had adverse events over grade 3 after treatment. The difference between each group was statistically significant ( $\mathrm{p}=0.027$ ). During CCRT with temozolomide plus adjuvant temozolomide, 11 patients $(27.5 \%$ ) showed nervous system disorder (including 3 hydrocephalus and 2 seizures), five patients $(12.5 \%)$ exhibited procedural complications (including 3 radiation recall reactions), three patients $(7.5 \%)$ experienced investigations (including 2 hematological toxicities), and so on. In the two patients $(5.0 \%)$ with reported hematological toxicity category, one patient had leukocytopenia and another patient had thrombocytopenia. For patients who underwent radiotherapy alone, there were four patients $(9.1 \%)$ with nervous system disorders, three patients (6.8\%) with musculoskeletal disorders, two patients $(4.5 \%)$ with psychiatric disorders, and so on. The distribution of the adverse events associated with the treatment is presented in Table 5. 
Table 5. Adverse event over grade 3

\begin{tabular}{lccc} 
& Total & $\begin{array}{c}\text { CCRT with temozolomide } \\
\text { plus adjuvant temozolomide }\end{array}$ & $\begin{array}{c}\text { Radiotherapy } \\
\text { alone }\end{array}$ \\
Gastrointestinal disorders & $3(3.8)$ & $3(7.5)$ & 0 \\
General disorders and administration site conditions & $1(1.3)$ & 0 & $1(2.3)$ \\
\hline Immune system disorders & $1(1.3)$ & $1(2.5)$ & 0 \\
Infections and infestations & $1(1.3)$ & $1(2.5)$ & $1(2.3)$ \\
Injury, poisoning, and procedural complications & $6(7.5)$ & $5(12.5)$ & 0 \\
Investigations & $3(3.8)$ & $3(7.5)$ & $1(2.3)$ \\
Metabolism and nutrition disorders & $4(5.0)$ & $3(7.5)$ & $3(6.8)$ \\
Musculoskeletal and connective tissue disorders & $4(5.0)$ & $3(7.5)$ & $4(9.1)$ \\
\hline Nervous system disorders & $15(18.8)$ & $11(27.5)$ & $2(4.5)$ \\
Psychiatric disorders & $2(2.5)$ & 0 & $1(2.3)$ \\
Renal and urinary disorders & $1(1.3)$ & 0 & $1(2.3)$ \\
\hline Vascular disorders & $1(1.3)$ & 0 & \\
\hline
\end{tabular}

Values are presented as number (\%). CCRT, concurrent chemoradiotherapy.

\section{Discussion}

This phase 2 trial of 84 patients demonstrated significant benefits for PFS with temozolomide during and after radiotherapy in patients with newly diagnosed WHO grade III glioma without the chromosome $1 \mathrm{p} / 19 \mathrm{q}$ co-deletion. Although there was no significant difference in OS between the two arms, our further analysis showed that age over 40 years was an independent risk factor for PFS (HR, 2.08) and that the presence of IDH1 mutation had a risk reduction for PFS (HR, 0.28) and OS (HR, 0.19).

The standard treatment protocol for WHO grade III gliomas has developed for many decades. Based on observations from previous studies, WHO grade III gliomas have been regarded as at least as chemosensitive as glioblastomas, and patients postoperatively treated with radiotherapy alone or chemotherapy alone fared worse than those treated with concurrent or adjuvant chemotherapy with radiotherapy $[16,17]$. Traditionally, anaplastic oligodendrogliomas are generally known to be chemosensitive and have had high rates of radiographic response in several trials after treatment with PCV [18]. However, chemotherapy with PCV has shown unacceptable toxicity, and the concurrent or adjuvant setting remains controversial. In the meantime, the efficacy found with adjuvant chemotherapy to radiotherapy for anaplastic astrocytoma, i.e., the $1 \mathrm{p} / 19 \mathrm{q}$ non-co-deleted anaplastic gliomas in the revised 2016 WHO classification, seemed more doubtful. Several studies found no significant survival benefits for adjuvant PCV [19-21]. However, CATNON trial identified adjuvant temozolomide prolongs survival for nonco-deleted anaplastic gliomas after radiotherapy [8]. In addition, our study showed the possibility that CCRT with temo- zolomide followed by adjuvant temozolomide has survival benefits for patients with WHO grade III gliomas.

This study adds to the literature for several reasons. This trial is the first investigator-initiated, multi-institutional, randomized trial for Korean adult patients with grade III glioma. For non-glioblastoma, compared to Whites, the incidence rate among Asians was substantially lower [22]. However, there have been fewer clinical trials only for Asian population. Especially, Koreans are one ethnic family, so long-term follow-up for unique ethnic group would be worth studying in terms of racial variation. And There should not be administrative, financial, and/or systemic difficulties in terms of performing clinical trials. We established a well-made system for this study and produced meaningful results. In addition, this trial included only patients with anaplastic gliomas without the chromosome $1 p / 19 q$ co-deletion. Since patients without $1 p / 19 q$ co-deletion gliomas had worse outcomes in the trials of PCV chemotherapy for anaplastic oligodendroglioma, we decided to take this approach $[7,18,23]$. Until now, there have been limited trials examining the efficacy and safety of temozolomide for patients with WHO grade III gliomas based on prospective molecular stratification. Furthermore, for patients with WHO grade III gliomas, the addition of temozolomide to radiotherapy, rather than PCV, is unusual [24]. Available data demonstrate with fair clarity that temozolomide is less toxic, easier to prescribe and less complicated for patients [25]. Although patients in this trial had $1 \mathrm{p} / 19 \mathrm{q}$ non-co-deletion anaplastic gliomas, temozolomide showed a clear benefit. Finally, we assessed not only the treatment outcome itself but also the consequence of the quality-of-life for patients who underwent the treatment protocol. The results of the quality-of-life will be reported separately. 
At the time of the beginning of this trial, genotypical characteristics were not considered in the WHO 2007 classification of glioma [26]. In 2009, mutations in IDH1 and IDH2 were reported to occur in $70 \%-80 \%$ of all grade II and III diffuse gliomas [14]. These mutations are associated with improved survival and are now the cornerstone of the revised WHO classification of gliomas $[15,27,28]$. Later, we incorporated analysis of the IDH1 mutation status and again demonstrated a significant survival benefit for patients with IDH1 mutation. However, in a subgroup analysis of MGMT promoter methylation and IDH1 mutation, there were no significant differences according to treatment modalities. This result could be due to the small sample size, or those markers might not have predictive value for benefit from CCRT with temozolomide. Further analysis with a large sample size is needed.

Our study has some limitations. (1) The number of patients remains relatively small compared with other trials for patients with adult gliomas. A future study with a larger sample size and more statistical power is necessary to validate findings from this study. (2) Only 2 years have passed since the last participant was enrolled in this study, and a more longterm follow-up is needed. In other trials for patients with WHO grade III gliomas, the survival curves begin to diverge 5-6 years after randomization. The lack of significance may have been due to limitations of follow-up periods, and the survival benefit of our regimen needs further analysis with long-term follow-ups. (3) As we sorted patients according to the WHO 2007 classification of glioma, there was a slight heterogeneity of the patient population. WHO grade III gliomas are now known to consist of heterogeneous groups of different histologic features, biological behaviors, and prognoses. As relevant molecular markers show their implications in response to therapy and survival, future studies should be based on discriminated molecular factors.

In conclusion, temozolomide during and after radiotherapy in Korean adult patients with newly diagnosed nonco-deleted WHO grade III gliomas showed an improved 2year PFS. Temozolomide, given its superior tolerability compared with PCV, showed acceptable adverse events. The survival benefit of this regimen requires further analysis with long-term follow-up at least more than 10 years. More prospective studies into the biology and prognostic significance of MGMT promoter methylation and other molecular mark- ers are also required.

\section{Conflicts of Interest}

Conflict of interest relevant to this article was not reported.

\section{Acknowledgments}

This study was supported by a grant from the National R\&D Program for Cancer Control, Ministry of Health and Welfare, Republic of Korea (1120320), and was partly supported by grant no. 02-2018018 from the Seoul National University Bundang Hospital Research Fund. The authors would like to thank Eun Jin Choi and Ho Nam Choi for their helpful efforts in coordinating all the research works. This study was coordinated by the Clinical Research Coordination Center, National Cancer Center, Korea using the web-based clinical research management platform (Velos).

\section{Author Details}

${ }^{1}$ Department of Neurosurgery, Seoul National University Bundang Hospital, Seoul National University College of Medicine, Seongnam, Departments of ${ }^{2}$ Internal Medicine and ${ }^{3}$ Neurosurgery, Seoul National University Hospital, Seoul National University College of Medicine, Seoul, ${ }^{4}$ Department of Neurosurgery, Severance Hospital, Yonsei University College of Medicine, Seoul, ${ }^{5}$ Department of Neurosurgery, Chonnam National University Hwasun Hospital, Hwasun, ${ }^{6}$ Department of Radiation Oncology, Keimyung University Dongsan Medical Center, Keimyung University School of Medicine, Daegu, 'Department of Neurosurgery, Samsung Medical Center, Sungkyunkwan University School of Medicine, Seoul, ${ }^{8}$ Department of Neurosurgery, Ajou University Hospital, Ajou University School of Medicine, Suwon, ${ }^{9}$ Department of Neuro-Oncology Clinic, Center for Specific Organs Cancer, National Cancer Center Hospital, National Cancer Center, Goyang, ${ }^{10}$ Department of Neurosurgery, Seoul St. Mary's Hospital, College of Medicine, The Catholic University of Korea, Seoul, ${ }^{11}$ Department of Neurosurgery, Inha University Hospital, Inha University School of Medicine, Incheon, ${ }^{12}$ Division of Cancer Epidemiology and Management, Research Institute, National Cancer Center, Goyang, Departments of ${ }^{13}$ Internal Medicine, ${ }^{14}$ Pathology, and ${ }^{15}$ Radiology, Seoul National University Bundang Hospital, Seoul National University College of Medicine, Seongnam, ${ }^{16}$ Department of Neurological Surgery, Asan Medical Center, University of Ulsan College of Medicine, Seoul, Korea

\section{References}

1. Izquierdo C, Joubert B, Ducray F. Anaplastic gliomas in adults: an update. Curr Opin Oncol. 2017;29:434-42.
2. Simonetti G, Gaviani P, Innocenti A, Botturi A, Lamperti E, Silvani A. Update on treatment strategies for anaplastic glio- 
ma: a review of literature. Neurol Sci. 2014;35:977-81.

3. Chibbaro S, Benvenuti L, Caprio A, Carnesecchi S, Pulera F, Faggionato $\mathrm{F}$, et al. Temozolomide as first-line agent in treating high-grade gliomas: phase II study. J Neurooncol. 2004;67: 77-81.

4. Sasaki H, Yoshida K. Treatment recommendations for adult patients with diffuse gliomas of grades II and III according to the new WHO classification in 2016. Neurol Med Chir (Tokyo). 2017;57:658-66.

5. Schiff D. Benefit with adjuvant chemotherapy in anaplastic astrocytoma. Lancet. 2017;390:1625-6.

6. Cairncross G, Wang M, Shaw E, Jenkins R, Brachman D, Buckner J, et al. Phase III trial of chemoradiotherapy for anaplastic oligodendroglioma: long-term results of RTOG 9402. J Clin Oncol. 2013;31:337-43.

7. van den Bent MJ, Brandes AA, Taphoorn MJ, Kros JM, Kouwenhoven MC, Delattre JY, et al. Adjuvant procarbazine, lomustine, and vincristine chemotherapy in newly diagnosed anaplastic oligodendroglioma: long-term follow-up of EORTC brain tumor group study 26951. J Clin Oncol. 2013;31:344-50.

8. van den Bent MJ, Baumert B, Erridge SC, Vogelbaum MA, Nowak AK, Sanson M, et al. Interim results from the CATNON trial (EORTC study 26053-22054) of treatment with concurrent and adjuvant temozolomide for $1 \mathrm{p} / 19 \mathrm{q}$ non-co-deleted anaplastic glioma: a phase 3, randomised, open-label intergroup study. Lancet. 2017;390:1645-53.

9. Stupp R, Mason WP, van den Bent MJ, Weller M, Fisher B, Taphoorn MJ, et al. Radiotherapy plus concomitant and adjuvant temozolomide for glioblastoma. N Engl J Med. 2005;352: 987-96.

10. Choi JW, Lee MM, Kim IA, Kim JH, Choe G, Kim CY. The outcomes of concomitant chemoradiotherapy followed by adjuvant chemotherapy with temozolomide for newly diagnosed high grade gliomas: the preliminary results of single center prospective study. J Korean Neurosurg Soc. 2008;44:222-7.

11. Kim YH, Park CK, Cho WH, Kim IA, Moon S, Choe G, et al. Temozolomide during and after radiation therapy for WHO grade III gliomas: preliminary report of a prospective multicenter study. J Neurooncol. 2011;103:503-12.

12. Jenkins RB, Curran W, Scott CB, Cairncross G. Pilot evaluation of $1 p$ and $19 q$ deletions in anaplastic oligodendrogliomas collected by a national cooperative cancer treatment group. Am J Clin Oncol. 2001;24:506-8.

13. Wen PY, Macdonald DR, Reardon DA, Cloughesy TF, Sorensen AG, Galanis E, et al. Updated response assessment criteria for high-grade gliomas: response assessment in neuro-oncology working group. J Clin Oncol. 2010;28:1963-72.

14. Yan H, Parsons DW, Jin G, McLendon R, Rasheed BA, Yuan $\mathrm{W}$, et al. IDH1 and IDH2 mutations in gliomas. N Engl J Med. 2009;360:765-73.

15. Louis DN, Perry A, Reifenberger G, von Deimling A, FigarellaBranger D, Cavenee WK, et al. The 2016 World Health Organization classification of tumors of the central nervous system: a summary. Acta Neuropathol. 2016;131:803-20.

16. Juratli TA, Lautenschlager T, Geiger KD, Pinzer T, Krause M,
Schackert G, et al. Radio-chemotherapy improves survival in IDH-mutant, 1p/19q non-codeleted secondary high-grade astrocytoma patients. J Neurooncol. 2015;124:197-205.

17. van den Bent MJ, Smits M, Kros JM, Chang SM. Diffuse infiltrating oligodendroglioma and astrocytoma. J Clin Oncol. 2017;35:2394-401.

18. Erdem-Eraslan L, Gravendeel LA, de Rooi J, Eilers PH, Idbaih A, Spliet WG, et al. Intrinsic molecular subtypes of glioma are prognostic and predict benefit from adjuvant procarbazine, lomustine, and vincristine chemotherapy in combination with other prognostic factors in anaplastic oligodendroglial brain tumors: a report from EORTC study 26951. J Clin Oncol. 2013; 31:328-36.

19. Medical Research Council Brain Tumor Working Party. Randomized trial of procarbazine, lomustine, and vincristine in the adjuvant treatment of high-grade astrocytoma: a Medical Research Council trial. J Clin Oncol. 2001;19:509-18.

20. Prados MD, Scott C, Curran WJ Jr, Nelson DF, Leibel S, Kramer S. Procarbazine, lomustine, and vincristine (PCV) chemotherapy for anaplastic astrocytoma: a retrospective review of radiation therapy oncology group protocols comparing survival with carmustine or PCV adjuvant chemotherapy. J Clin Oncol. 1999;17:3389-95.

21. Prados MD, Seiferheld W, Sandler HM, Buckner JC, Phillips T, Schultz C, et al. Phase III randomized study of radiotherapy plus procarbazine, lomustine, and vincristine with or without BUdR for treatment of anaplastic astrocytoma: final report of RTOG 9404. Int J Radiat Oncol Biol Phys. 2004;58:1147-52.

22. Dubrow R, Darefsky AS. Demographic variation in incidence of adult glioma by subtype, United States, 1992-2007. BMC Cancer. 2011;11:325.

23. Cairncross JG, Wang M, Jenkins RB, Shaw EG, Giannini C, Brachman DG, et al. Benefit from procarbazine, lomustine, and vincristine in oligodendroglial tumors is associated with mutation of IDH. J Clin Oncol. 2014;32:783-90.

24. Tham CK, See SJ, Tan SH, Lim KH, Ng WH, Thomas J, et al. Combined temozolomide and radiation as an initial treatment for anaplastic glioma. Asia Pac J Clin Oncol. 2013;9:220-5.

25. Lassman AB. Procarbazine, lomustine and vincristine or temozolomide: which is the better regimen? CNS Oncol. 2015;4: 341-6.

26. Louis DN, Ohgaki H, Wiestler OD, Cavenee WK, Burger PC, Jouvet A, et al. The 2007 WHO classification of tumours of the central nervous system. Acta Neuropathol. 2007;114:97-109.

27. van den Bent MJ, Dubbink HJ, Marie Y, Brandes AA, Taphoorn MJ, Wesseling $\mathrm{P}$, et al. IDH1 and IDH2 mutations are prognostic but not predictive for outcome in anaplastic oligodendroglial tumors: a report of the European Organization for Research and Treatment of Cancer Brain Tumor Group. Clin Cancer Res. 2010;16:1597-604.

28. Sanson M, Marie Y, Paris S, Idbaih A, Laffaire J, Ducray F, et al. Isocitrate dehydrogenase 1 codon 132 mutation is an important prognostic biomarker in gliomas. J Clin Oncol. 2009;27:4150-4. 